\title{
Effects of perceptual training of sequenced line movements
}

\author{
RAY EBERTS \\ Purdue University, West Lafayette, Indiana \\ and \\ WALTER SCHNEIDER \\ University of Pittsburgh, Pittsburgh, Pennsylvania
}

\begin{abstract}
The effects of consistent training on sequential stimuli were examined in a series of six experiments. A stimulus consisted of three line segments which occurred one after another such that each line segment's orientation and position on the visual channel could be different from the other lines of the stimulus. Subjects practiced these sequences in search and detection tasks for $17 \mathrm{~h}$ in both varied mapping (VM) and consistent mapping (CM) conditions. Subsequent experiments compared performance on the two practice conditions with results of previous experiments which used letters and digits as stimuli. The results indicated that the CM stimuli required less attention than the VM stimuli. The differences seen, however, were smaller than those found in previous experiments with letter and digit stimuli. Large differences between the CM and VM groups were found when the individual line segments of the sequential stimuli were manipulated. Shortening and lengthening the line durations had little effect on CM stimuli and large effects on VM stimuli. When the line segments were overlapped with each other, subjects indicated, in a subjective ratings task, that the CM and VM stimuli were perceived differently. Finally, presenting rotated versions of the trained stimuli destroyed any performance advantages the CM stimuli had over the VM stimuli.
\end{abstract}

In the LaBerge (1973) theory of information processing, memory is conceptualized as a network of nodes. When sensory stimulation occurs, the information is analyzed by feature detectors. Output from selected groups of detectors then feed into a higher level pattern code. LaBerge (1976) found that, with extended practice, the processing of novel characters became less sensitive to attentional manipulations. Shiffrin and Schneider (Schneider \& Shiffrin, 1977; Shiffrin \& Schneider, 1977) examined attentional load in detection and divided attention tasks. The theoretical perspective taken by Shiffrin and Schneider (1977) was similar to that of the LaBerge theory. Briefly, they conceptualized memory as a large collection of nodes that could be interrelated and associated to each other through learning and practice (see Shiffrin \& Schneider, 1977, pp. 155-164). When a stimulus is perceived, one of two processes, or a combination of the two processes, is utilized. One of the processes, an automatic process, utilizes a relatively permanent set of associations in long-term storage that is built up through hours of practice. This automatic process occurs when the sequence of nodes becomes active in response to a particular input configuration. On the other hand, a con-

This project was supported in part by funds from Office of Naval Research Contract N00014-86-K-0107, W. Schneider, Principal Investigator. Correspondence should be directed to R. Eberts, School of Industrial Engineering, Purdue University, West Lafayette, IN 47907. trolled process utilizes a temporary sequence of nodes activated under control and through attention of the subject. This process can be set up, altered, and applied with relative ease to novel configurations (for a detailed model, see Schneider, 1985).

An important contribution of the Shiffrin and Schneider work was to show that the development of these two processes was dependent on the kind of training given. An automatic process develops only if the stimuli are consistently mapped (see Schneider \& Fisk, 1982b). In a consistently mapped situation, a target is always a target whenever it occurs and is never a distractor. A controlled process is used in varied mapping situations in which a particular stimulus can be a target on one trial and a distractor on another. Even after long hours of training, a stimulus will not be processed automatically if it has been trained in a varied mapping situation (Schneider \& Shiffrin, 1977). Automatic processing is characterized by low resource load and an inability to control the processing (see Jonides, Naveh-Benjamin, \& Palmer, 1985). Automatic processing has been developed as a result of consistent training for character search (e.g., Schneider \& Shiffrin, 1977; Schneider \& Fisk, 1982a, 1982b), semantic search (Fisk \& Schneider, 1983; Schneider \& Fisk, 1984), and auditory search (Poltock, Lansman, \& Hunt, 1981).

The following experiments examined automaticity and the effects of consistent and varied training to a different 
stimulus set: stimuli composed of three line segments appearing one after the other. These stimuli will be referred to as sequences. The experiments examined whether a consistent sequence of features could trigger an automatic response. Many of the skilled behaviors exhibited in the real world depend on the visual perception of events that change both spatially and temporally as do the sequences. In driving a car, it is critical that an object moving into the path of the car attract attention. Any kind of hand-toeye coordination, such a catching a ball or throwing a ball to a moving target, requires a response to stimuli that move over time. Visual sequential stimuli have been studied in the past to determine how discrete events are combined to appear as movement (see Graham, 1965; Kahneman, 1967; Kolers, 1964; Restle, 1979; Wertheimer, 1912), to study characteristics of forward and backward masking (see Bushbaum \& Mayzner, 1969; Fehrer \& Raab, 1962), and to study the effects of selective attention on motion patterns (Neisser \& Becklen, 1975). Evidence indicates that auditory stimuli, which are sequential by nature, can be processed automatically (Ostry, Moray, \& Marks, 1976).

The detection of sequential stimuli were examined in the following six experiments. The sequences were composed of three equal-length lines which occurred one after the other. The lines that composed the sequence could vary in orientation and position. For these stimuli, a feature can be defined as a simple unit, a straight line, and it can also be defined as occurring at a certain time in the stimulus. In the first experiment, subjects were trained for over $17 \mathrm{~h}$ on two groups of stimuli: stimuli that were consistently mapped (the CM stimulus group) and stimuli that were variably mapped (the VM stimulus group). The next two experiments (Experiments 2 and 3 ) examined the attentional demands required to detect stimuli from the two stimulus groups. Results from Experiments 1 to 3 were compared with the results of earlier research, which employed similar manipulations and used letters and digits as stimuli. In Experiments 4 and 5, the sequences were altered by changing the line duration and the line overlap. These experiments were performed to try to determine how the processing of the sequences was different from the processing of letters and digits. Experiment 6 altered the sequences by rotating them in order to investigate the transfer of the practice effects to stimuli that were changed in orientation.

\section{GENERAL METHOD}

\section{Subjects}

The subjects were 8 University of Illinois undergraduates ( 3 male), who were paid for their participation. Because of an illness and later time conflicts, 1 subject was forced to drop out after the first experiment. Her data will not be reported. The remaining 7 subjects participated in all the experiments. All were right-handed, and all had normal or corrected-to-normal vision.

\section{Apparatus}

The experiments were controlled by a PDP-11/34 digital computer. Displays appeared on Tektronix 604 or 620 cathode ray rubes, which were equipped with P-31 phosphor with a refresh rate of
$10 \mathrm{msec}$. The scope consisted of a $1,024 \times 1,024$ unit matrix, such that each cell in the matrix could be controlled individually. Four self-paced subjects, separated from each other by partitions, could be run at a time.

\section{Stimuli}

A four-channel detection task was used. To eliminate eye movements, the target occurred on one of the four channels with distractors occurring on the other three. The whole display subtended about $2.8^{\circ}$ of visual angle. Each channel was situated at the four corners of an imaginary square separated by $1^{\circ}$ of visual angle so that lateral masking would be minimal (Eriksen \& Eriksen, 1974). A channel subtended about $0.9^{\circ}$ of visual angle both horizontally and vertically.

The sequences were composed of 3 line segments that produced a complex noncontinuous movement. Each line segment subtended about $0.6^{\circ}$ of visual angle; 28 line segments, differing in location and orientation, were used to generate the sequences. The duration of each line of the three-line sequences was $100 \mathrm{msec}$ with an interstimulus interval (ISI) of $0 \mathrm{msec}$. The ISI and the line duration were chosen from work done by the Gestaltists and others (see DeSilva, 1926; Graham, 1965; Kahneman \& Wolman, 1970) to enhance the motion characteristics of the sequences so that individual sequences could be perceived as a stimulus rather than as a series of three separate lines.

For a pilot study, 35 sequences were composed pseudo-randomly. From these 35 , the sequences of intermediate difficulty, in terms of percent correct (see Eberts, 1979), were used in the following experiments. These were further divided into sets of $A$ and $B$, which were equated for difficulty (see Figure 1 for the sequences used). For half the subjects, Set A was designated the CM set and Set B was the VM set. The other subjects had the opposite assignment. Each individual subject was assigned one sequence from the $\mathrm{CM}$ set, designated the CM target sequence, which was always the target in the CM condition for that subject throughout the whole series of experiments. In the VM condition, the target sequence was chosen randomly from one of the eight sequences in the VM set before each trial. These eight targets will be called VM targets.

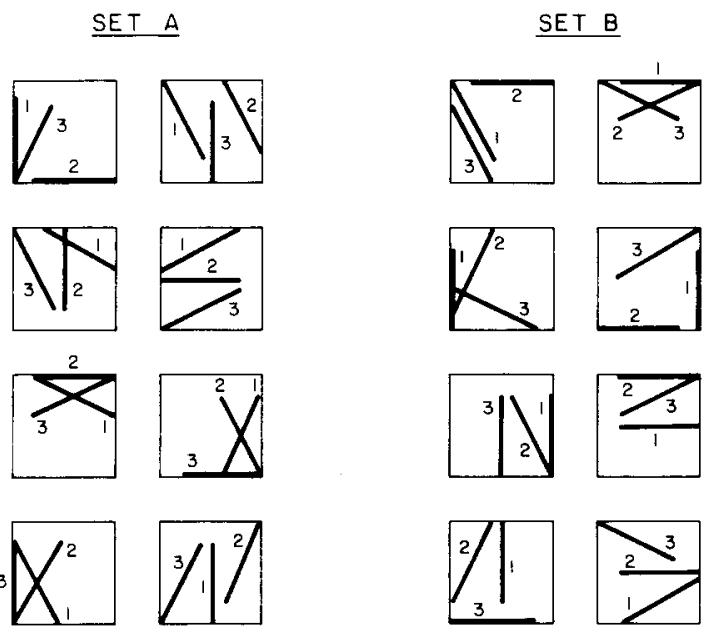

Figure 1. The sequences used. The order of occurrences of the line segments is signified by the number. The sequences were equated for difficulty in a pilot study and divided into two sets, $A$ and $B$. One group of subjects had set $A$ as $C M$ sequences and set $B$ as VM sequences. The other group had the opposite assignment. The sequences for each set are ordered by ease of identification, as determined by the pilot study, with the easiest at the top and the most difficult at the bottom. The middle four sequences of each set were chosen as the $C M$ sequences. 


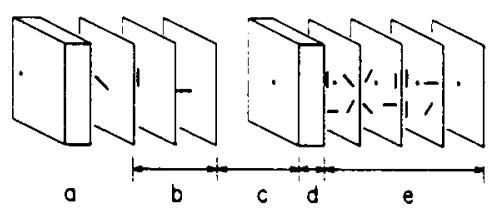

Figure 2. An example of a trial in the one-sequence procedure of Experiment 1 and following experiments: (a) 500 -msec focus dot at the start of the orientation, (b) the presentation of the orientation sequence that specifies the target, (c) a 1-sec pause, (d) the beginning of the experimental trial with a 500 -msec focus dot, and (e) the trial display of four sequences followed by random-dot masks. The target sequence appears in the upper right position in this example.

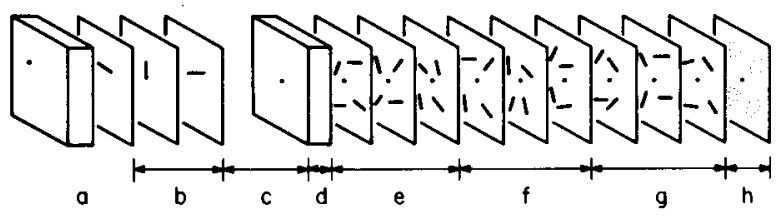

Figure 3. An example of a trial in the three-sequence procedure of Experiment 1: (a) 500 -msec focus dot at the start of the orientation, (b) the presentation of the orientation sequence that specifies the target, (c) a 1-sec pause, (d) the beginning of the experimental trial with a 500-msec focus dot, (e) the first group of sequences, (f) the second group of sequences which must contain the target sequence, (g) the third group of sequences, and (h) random-dot masks. The target sequence appears in the lower right position in the second group of sequences in this example.
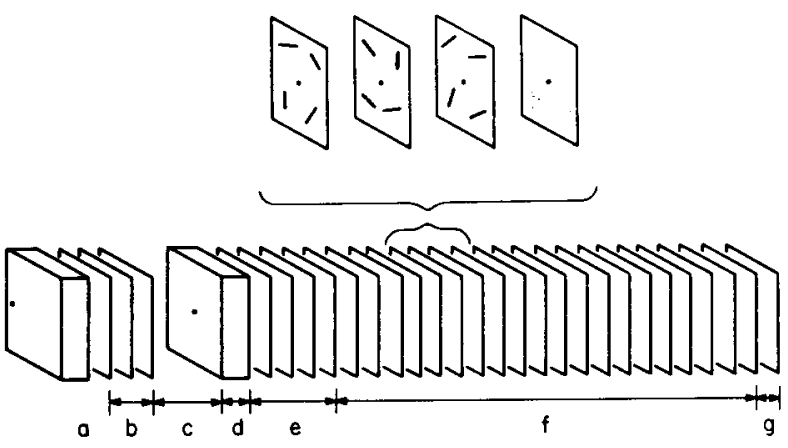

Figure 4. An example of the six-sequence procedure used in Experiment 1: (a) a 500-msec focus dot for the orientation target sequence, (b) the presentation of the orientation sequence that specifies the target, (c) a 1-sec pause, (d) the beginning of the experimental trial with a 500-msec focus dot, (e) the first of the six groups of sequences (a blowup of the third group of sequences is shown), (f) the remaining groups of sequences, and (g) the final random-dot mask. The target cannot occur in the first or last groups of sequences.

Depending on the experiment, the trial display could be one of three types: a one-sequence, three-sequence, or six-sequence display. In the one-sequence display (see Figure 2), single sequences, followed by random-dot mask patterns in each of the four channels, were displayed. The target sequence of the particular subject was displayed in one of the four channels, with randomly chosen distractor sequences occupying the remaining three channels. In Figure 2, the target appears in the upper right corner of the display. A three-sequence display (see Figure 3) was composed of three sequences in each of the four channels. The target sequence ap- peared randomly in one of the four channels as the second of the three sequences (It could appear only in the section labeled " $f$ " in Figure 3). Distractor sequences occupied the other positions and were chosen randomly from the distractor set, with the restriction that the same sequence could not occur consecutively. In Figure 3, the target occurs in the lower right corner for the second sequence. A six-sequence display (see Figure 4) was composed of six sequences followed by random dot masks in each of the four channels (these sequences could appear in the sections labeled " $e$ " and " $f$ " in Figure 4). The target sequence occurred randomly on one of the four channels as the second, third, fourth, or fifth sequence on that channel. In Figure 4, the target appears as the third sequence in the upper right position. All other sequences were chosen randomly from the distractor set so that the same sequence could not appear consecutively on the same channel.

\section{Procedure}

To start a trial, subjects pushed a button in the upper left corner of a response box with the left index finger. First, a 500-msec focus dot appeared to the right of the center of the screen. On the offset of the focus dot, the three-line target sequence appeared.

One second later, the trial display began. Directly in the middle of the screen, $2.2^{\circ}$ to the right of the position of the orientation target sequence, a 500-msec focus dot appeared. Depending on the experiment, the trial display could be the one-sequence, threesequence, or six-sequence display depicted in Figures 2-4. One, and only one, of the sequences in the total display was the target sequence that the subjects saw at the beginning of the trial. After the trial display, random dot masks appeared on all four channels. The subjects were instructed to push one of the four buttons (corresponding to the position of the target on the screen) on the response box with the right index finger; they were instructed to guess if they were uncertain of the position of the target. They were allowed $4 \mathrm{sec}$ for a response.

If the subjects made an incorrect response, an $X$ appeared in the correct position on the screen for $500 \mathrm{msec}$ and a 500 -msec error tone sounded over the subjects' headset. After each trial, the percentage of trials correct for the current block appeared as a number in the lower left corner of the display. The score remained on the screen for a maximum of $30 \mathrm{sec}$. Any time during this period the subjects could start the next trial by pushing the upper left button on the response box. At the end of a block of trials, the subject's percentage score for the entire block appeared in the middle of the screen. Each session lasted $50 \mathrm{~min}$.

\section{EXPERIMENT 1 TRAINING}

In this experiment, subjects were trained for over $17 \mathrm{~h}$ on their CM and VM sequences. To reduce the ceiling effects and to make the task more difficult and challenging, training proceeded from the one-sequence display to the three-sequence display and then finally to the sixsequence display. The purpose of this experiment was to compare the results with sequences with the results of experimentation that had used letters as stimuli (e.g., Shiffrin \& Schneider, 1977, Experiment 1). In the Shiffrin and Schneider experiment, the CM performance was always higher than the VM performance and improved at a faster rate than the VM performance. The present experiment tested the same aspects of the data for the sequence stimuli.

\section{Method}

All three trial displays were used during the course of this experiment. The type of practice, either CM or VM, was manipu- 
lated as a between-block variable. Within a block of trials in the VM condition, each VM target appeared eight times for a total of 64 trials. In the CM condition, all 64 trials of the block were spent searching for the single $\mathrm{CM}$ target. The order of occurrence of $\mathrm{CM}$ and VM blocks was randomized within groups of two. A CM and VM block together will be termed a replication

The subjects were first trained in a one-sequence procedure for a total of 25 replications which lasted about $7 \mathrm{~h}$. All subjects were then switched to the three-sequence display and were trained on 20 replications for approximately $6 \mathrm{~h}$. Next, they were switched to the six-sequence procedure for 15 replications, which took approximately $4 \mathrm{~h}$. During the whole training procedure, the subjects received 3,840 trials in the $C M$ condition and 3,840 trials in the VM condition.

\section{Results and Discussion}

Scores were corrected for guessing by subtracting onethird the probability of a miss from the probability of a hit. In Figure 5, each point represents the average of five replications over all three display types. The sequences were successfully equated for difficulty, as evidenced by the nonsignificant difference between the two stimulus groups in the first replication $[t(6)=1.04]$. Because three separate procedures were used (one-, three-, and sixsequence displays), three separate analyses of variance (ANOVAs) were used to analyze the data. For all three analyses, a three-way $(\mathrm{CM} / \mathrm{VM}$ stimuli $\times$ replications $\times$ subjects) repeated measures ANOVA, with replications and $\mathrm{CM} / \mathrm{VM}$ stimuli the repeated measures, was performed on the data.

For the one-sequence display (Replications 1-25), CM stimuli were identified better than the VM stimuli $[F(1,6)$ $=8.87, p<.025]$. As the subjects had more practice on the sequences, performance improved $[F(24,144)=$ 26.73, $p<.001]$. The interaction between replications and the two different kinds of stimuli was also significant $[F(14,24)=1.78, p<.025]$. For the three-sequence displays (Replications 26-45), CM sequences were still identified better than the VM sequences $[F(1,6)=14.73$, $p<.01]$, and the interaction between those two factors was significant $[F(19,114)=39.2, p<.001]$. For the six-sequence display (Replications 46-60), CM stimuli were identified better than VM stimuli $[F(1,6)=39.2$, $p<.001]$, performance improved with practice $[F(14,84)$ $=3.42, p<.001]$, and the interaction between the two factors was significant $[F(14,84)=2.38, p<.01]$.

Throughout the experiment, the subjects became better at identifying the sequences. The amount of improvement was very high for the one-sequence displays, but tended to slow down some by the time the subjects were given the six-sequence displays. For all three display types, the subjects could always identify the CM sequences better than the VM sequences. The significant interactions indicated that the subjects improved faster on

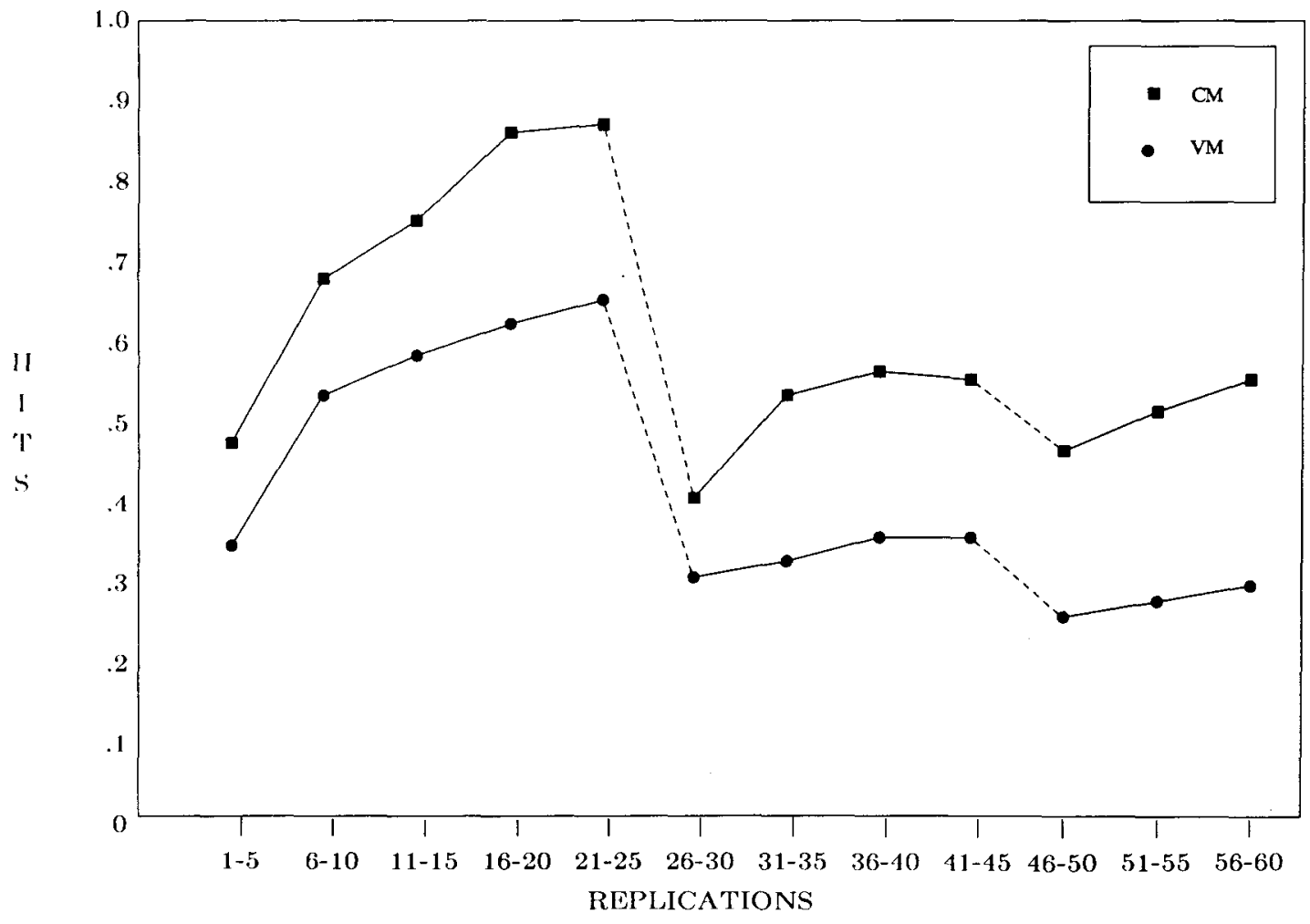

Figure 5. The proportion of trials identified correctly as a function of practice from Experiment 1 . The hits are corrected for guessing. A one-sequence procedure is used through replication 25 , a three-sequence display is used through replication 45 , and a six-sequence display is used through replication 60 . 
the $\mathrm{CM}$ sequences than they did on the VM sequences. These data were similar to those of Shiffrin and Schneider (1977, Experiment 1), using letters, when they also showed that CM performance was better than VM performance and that $\mathrm{CM}$ performance improved at a faster rate than VM performance. At the end of this training, the subjects were well trained in identifying the sequences.

\section{EXPERIMENT 2 VARIABLE NUMBER OF CHANNELS}

In this experiment, the number of channels was varied between one, two, and four channels. The resulting function that relates accuracy to the number of channels provides an indication of the effect that the different kinds of practice had on the ability to share attention between the channels. The number of channels manipulation corresponds directly to the frame size manipulations of Schneider and Shiffrin (1977, Experiment 2). In varying the frame size, the number of positions in which distractors and targets could occur was varied between one, two, and four. In their experiments, Schneider and Shiffrin found that changes in the frame size affected VM performance (i.e., performance decreased as frame size increased) but had little or no effect on CM performance.

\section{Method}

A six-sequence procedure, similar to that illustrated in Figure 4, was used. However, the separating masks between sequences were not present. The practice condition, $\mathrm{CM}$ or VM, and the number of channels (one, two, or four) were both between-block variables. In the one-channel condition, the single channel containing the sequences was chosen randomly before each trial. The other three channels were filled with random dot masks and remained constant throughout the trial display. In the two-channel condition, one of the two possible diagonals was chosen randomly before each trial to contain the sequences. The other two positions of the off-diagonal were filled with random dot masks. In the four-channel condition, all four channels were filled with sequences.

Each block contained 64 trials. The target occurred on half the trials. The subjects were instructed to rest the index and the middle fingers of their right hands on two buttons of the response box. Four of the subjects were told to push the rightmost button with the middle finger if a target occurred and the leftmost button with the index finger if no target occurred. The remaining subjects had the opposite button assignment. The subjects participated in 18 blocks of trials altogether, 3 blocks for each of the three channel sizes for the two practice conditions. This experiment lasted approximately $2 \mathrm{~h}$.

\section{Results and Discussion}

The data are plotted in Figure 6 in terms of the $A^{\prime}$ scores. $A^{\prime}$ is a measure of sensitivity similar to $d^{\prime}$. It is, however, a distribution-free measure which is desirable (Craig, 1979; Norman, 1964) when the false-alarm rate is low, as it is in these data for some of the subjects. For the $A^{\prime}$ score, 1.0 represents perfect detection and .50 represents random guessing. The $A^{\prime}$ scores, after an arcsin transform, were analyzed by a repeated measures three-way ANOVA with subjects the random factor. Overall performance decreased as the number of chan-

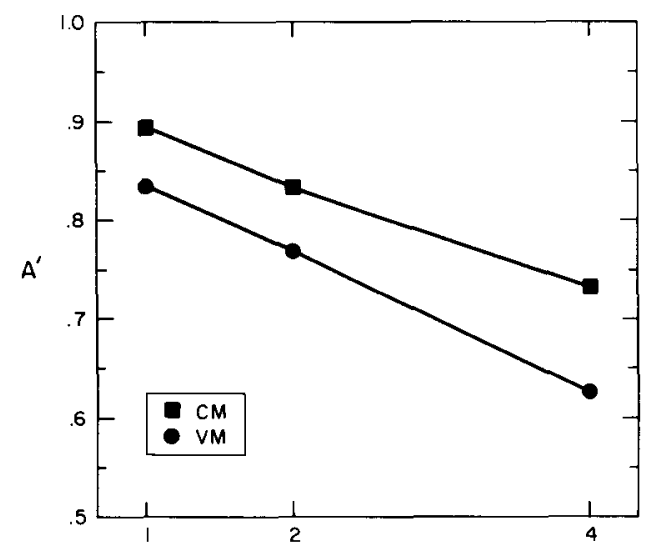

NUMBER OF CHANNELS

Figure 6. $A^{\prime}$ as a function of number of channels for $\mathrm{CM}$ and $\mathrm{VM}$ sequences from Experiment 2.

nels was increased $[F(1,6)=39.96, p<.001]$. The $C M$ sequences were identified as marginally better than the VM sequences $[F(1,6)=5.30, p<.06]$. The interaction between channel size and the type of practice was nonsignificant.

The lack of an interaction indicated that varying the number of channels affected CM and VM sequences about equally. This result is different from that found by Schneider and Shiffrin (1977), where frame size had no effect on the CM stimuli but had an effect on the VM stimuli. In the present experiment, CM sequences were better identified overall, but they did not exhibit the same characteristics as those seen for the letter and digit stimuli in the Schneider and Shiffrin experiment.

\section{EXPERIMENT 3 DUAL SEARCH}

Schneider and Fisk (1982a), using a dual-task paradigm, found that the detection of consistently mapped visual characters required little mental effort for identification after training. Mental effort was evaluated, in one condition, by having the subject search for both a CM and VM target on the same trial. Performance in this dual task was then compared with that in the search for a single $C M$ or VM target. Schneider and Fisk found that even when subjects were instructed to protect their VM performance in the dual task, by emphasizing detection of the VM target so that VM dual performance was the same as VM performance on the single-task trials, the subjects could still detect the $\mathrm{CM}$ targets with little deficit in performance relative to that in the $\mathrm{CM}$ single condition. Because $\mathrm{CM}$ performance was still high even when no attention was allocated to the detection of these stimuli, Schneider and Fisk concluded that CM detection required little or no attentional resources for detection. This prior research used letters and digits as stimuli; the present experiment used the sequence stimuli on which subjects had been trained extensively. 


\section{Method}

A one-sequence procedure similar to that depicted in Figure 2 was used. This experiment contained four conditions: (1) a CMVM condition in which subjects were required to dual search for both a CM and a VM target; (2) a VMVM condition in which subjects were required to dual search for two VM targets; (3) a CM condition in which subjects were required to search for their CM target; and (4) a VM condition in which subjects searched for a single VM target. The procedures for the CM and VM conditions were exactly the same as the first part of Experiment 1. In the VMVM condition, the subjects were given both target sequences during the orientation part of the trial. The two VM target sequences, each preceded by a $500-\mathrm{msec}$ focus dot, occurred one after the other in the upper right corner of the display. The orientation sequences were separated from each other by $500 \mathrm{msec}$. The trial display always contained one, and only one, of the target sequences; half of the trials within a block of trials contained the first sequence that was displayed. The subjects were told to place equal importance on finding either of the two targets.

The CMVM condition was similar to the VMVM condition except that, in the orientation part of the trial in which the first target VM sequence had occurred earlier, an 800 -msec focus dot appeared, instead of the CM target sequence; the focus dot indicated to the subjects that they were in the CMVM condition. By this time, the subjects did not have to be reminded what their CM target sequence looked like. The subjects were instructed to concentrate all of their attention on searching for the VM target sequence and not to concentrate on searching for the $\mathrm{CM}$ target. During a block of trials, the VM target occurred on $75 \%$ of the trials and the CM target occurred on the remaining trials; the subjects were aware of this probability. By protecting the VM trials, performance on the $\mathrm{CM}$ sequences should give an indication of the amount of effort required to identify the CM sequences. The procedure for this experiment was exactly the same as that for Experiments $2 \mathrm{C}$ and 3 from the Schneider and Fisk (1982a) research, except that this experiment contained a VMVM condition and the Schneider and Fisk experiments did not.

The subjects received 12 blocks of trials, three replications of each of the four conditions. This experiment lasted approximately $11 \frac{1}{2} \mathrm{~h}$.

\section{Results and Discussion}

The subjects followed instructions by protecting their VM peformance from the VM single condition (.75) to the VM performance in the dual CMVM condition (.73). This difference was nonsignificant.

A repeated measures three-way ANOVA, with subjects as the random factor, was performed on the corrected scores after an arcsin transformation (see Figure 7). A significant decrement in performance occurred when the subjects were required to search for two targets $[F(1,6)$ $=13.19, p<.025]$. Although the $\mathrm{CM}$ stimuli were detected better overall than the VM stimuli, this difference was nonsignificant $[F(1,6)=3.10]$. The interaction between number of targets and stimulus group (CM or VM) was also nonsignificant.

The averaged data reported above may be misleading because 2 of the subjects had CM dual target scores that were very different from those of the other 5 subjects. These 2 subjects had CM uncorrected detection scores in the two-target condition that were .23 and .27 ; the lowest score among the other subjects was .78. Since these 2 subjects were performing at chance detection, they may have set a $\beta$ criterion that was different from that of the other

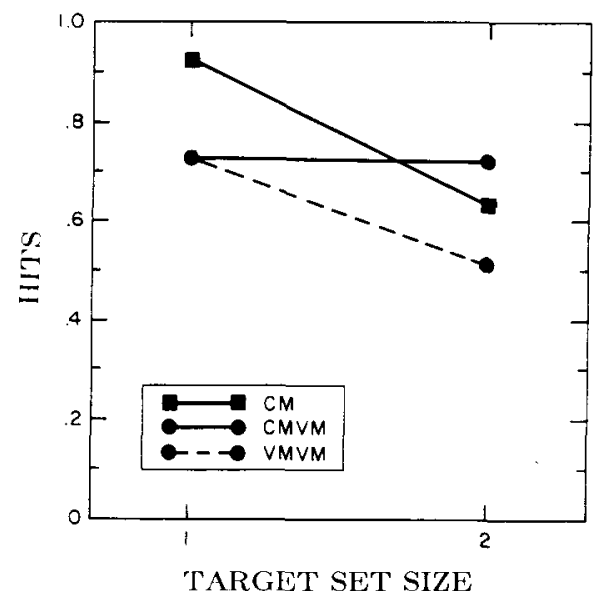

Figure 7. The proportion of trials identified correctly as a function of the target size from Experiment 3. The scores are corrected for guessing.

subjects (Schneider \& Fisk, 1982a, found large $\beta$ criterion shifts between the subjects). An analysis performed without the 2 outlying subjects still found differences due to the memory set size $[F(1,4)=15.0, p<.025]$ and also found large $\mathrm{CM} / \mathrm{VM}$ differences $[F(1,4)=135.2$, $p<.001]$. Also, the interaction between the two factors approached significance $[F(1,4)=4.71, p<.10]$. These results for the 5 subjects were not due to a tradeoff in focusing attention on the CM stimuli because, as found for the subjects as a group, the difference between VM single (.80) and VM dual (.76) was nonsignificant.

An important purpose of this experiment was to compare its results with those of the Schneider and Fisk (1982a) experiments, which used letters and digits as stimuli. Table 1 compares the performance of subjects in Schneider and Fisk's Experiments $2 \mathrm{C}$ and 3 with the performance of all subjects in this experiment and with that of the 5 subjects whose CM performance did not drop to chance levels. The procedures of all these experiments were the same except that Schneider and Fisk did not include the VMVM condition and they manipulated the frame time of the letter or digit stimulus. The difference between $\mathrm{CM}$ dual and CM single performance, the important indication of the attentional resources needed for the CM stimulus, was greater $(.35)$ than any of the differences found in the Schneider and Fisk experiments (the highest difference was .33 for the shortest frame time of $90 \mathrm{msec}$ in Experiment 3). The CM single/dual difference for the 5 subjects, however, fell well within the range of values obtained from the Schneider and Fisk experiments.

In some ways, the results from the sequences were similar to those found in the Schneider and Fisk (1982a) experiments, which used letters and digits as stimuli. Performance of individual subjects certainly overlapped the performance of individual subjects in the Schneider and Fisk experiments; in fact, many of the subjects in the present experiment exceeded the performance levels of 
Table 1

Comparison of Sequence Results to Letter and Digit Results from Schneider and Fisk (1982a)

\begin{tabular}{lcccccc}
\hline Experiment & $\begin{array}{c}\text { Frame } \\
\text { Time }(\mathrm{msec})\end{array}$ & $\begin{array}{c}\text { Single } \\
\text { VM }\end{array}$ & $\begin{array}{c}\text { Dual } \\
\text { VM }\end{array}$ & $\begin{array}{c}\text { Single } \\
\text { CM }\end{array}$ & $\begin{array}{c}\text { Dual } \\
\text { CM }\end{array}$ & $\begin{array}{c}\text { Difference } \\
\text { Single, Dual CM }\end{array}$ \\
\hline ES, All Subjects & - & .66 & .67 & .97 & .62 & .35 \\
ES, 5 Subjects & - & .80 & .76 & .96 & .84 & .12 \\
SF 2C & 90 & .41 & .38 & .65 & .41 & .24 \\
& 130 & .48 & .59 & .94 & .78 & .16 \\
& 180 & .65 & .66 & .96 & .86 & .10 \\
SF 3 & 90 & .37 & .37 & .63 & .30 & .33 \\
& 130 & .60 & .54 & .88 & .76 & .12 \\
& 180 & .65 & .62 & .89 & .86 & .03 \\
\hline
\end{tabular}

Note-ES refers to Eberts and Schneider, this report; SF 2C refers to Schneider and Fisk, 1982a, Experiment 2C; and SF 3 refers to Schneider and Fisk, 1982a, Experiment 3.

Schneider and Fisk's subjects. The VMVM condition allowed us to compare CMVM dual performance with VMVM dual performance, a comparison that was not made in the Schneider and Fisk experiments. The VMVM condition was not entirely comparable to the CMVM condition, however, because, in the VMVM condition, the subjects were instructed to divide their attention between the two stimuli whereas in the CMVM condition the subjects were instructed to place all their attention on detection of the VM stimulus. A comparison of these two conditions shows that VM and CM performance dropped at the same rate when the subjects moved from the single to the dual task. The CM single/dual task differences show that some decrement occurred when the switch to the dual task was made, and that, for 5 of the 7 subjects, this decrement is comparable to that found with Schneider and Fisk's subjects. The CM targets could be identified at a fairly high rate by most of the subjects even when they were instructed not to allocate attention to these stimuli.

\section{EXPERIMENT 4 VARIABLE LINE DURATION}

In this experiment, transfer stimuli were devised by increasing the duration of the lines that were used in the sequential stimuli and holding constant other characteristics of the stimulus. In particular, the duration of the line segments in the sequences (during training the duration was $100 \mathrm{msec}$ ) was varied from 70 to $220 \mathrm{msec}$.

This experiment was designed to evaluate the memory and attentional requirements needed to process $\mathrm{CM}$ and VM stimuli. Experiment 3 had shown that the CM stimulus could be identified accurately by most subjects even if the subjects paid little attention to it. The VM stimulus required more attentional resources. By varying the amount of time that any of the features of the stimulus remain on the screen, the total amount of attentional resources devoted to a particular feature can be manipulated. Also, maintaining the target sequence in memory may require attentional resources. Unlike letter and digit stimuli, the features of the sequences occurred at different times. To identify the sequence, the subject would have to remember the first and second lines of the sequence at the time the third line occurred. If the decay time for memory traces for CM and VM are different or the attentional demands to keep the memory trace updated are different, then these differences would be reflected in the ability to identify the CM and VM sequences.

\section{Method}

The three-sequence procedure depicted in Figure 3 was used. The independent variable of importance was line duration, a betweentrial variable. On a trial, the line segments of the sequences could have six possible durations: $70,100,130,160,190$, and $220 \mathrm{msec}$. The target orientation sequence had the same line duration as that used for the trial display. Each block contained 60 trials. Within the block, the 100 -msec duration occurred 30 times and the other five durations occurred on 5 trials each. The manipulation of the type of practice, either CM or VM, was a between-block variable. The subjects participated in a total of 16 blocks $(8 \mathrm{CM}$ blocks and 8 VM blocks).

\section{Results and Discussion}

A repeated measures three-way ANOVA, with subjects as the random factor, was performed on the corrected percentage scores after an arcsin transformation (see Figure 8). CM stimuli were detected better than VM stimuli

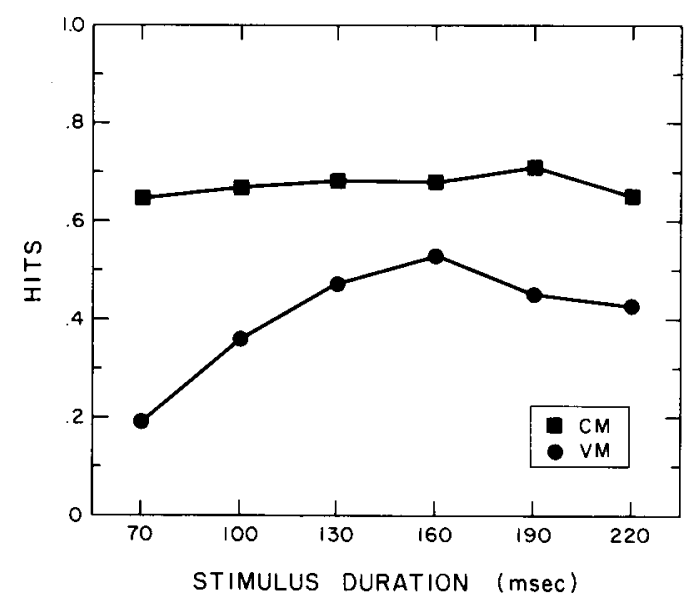

Figure 8. The proportion of trials identified correctly as a function of the line duration from Experiment 4 . The hits are corrected for guessing. 
$[F(1,6)=10.82, p<.05]$, and, generally, performance improved as the stimulus duration increased $[F(5,30)=$ $7.33, p<.001]$. More importantly, the interaction between stimulus group and line duration was significant $[F(5,30)=6.70, p<.001]$. CM performance stays fairly constant, with a $4 \%$ increase up to the 190 -msec duration level; VM performance improves $21 \%$ up to the 160 msec level, and then drops off.

The flat function relating performance to line duration in the CM condition indicates that performance was relatively unaffected by changes that occurred to the line durations for $\mathrm{CM}$ stimuli. In the VM condition, however, performance was affected by this manipulation. When line duration was decreased, performance got worse. When line duration was increased, performance got better. Finally, when line duration was at its longest, performance dropped off.

The inverted-U-shaped curve for the VM stimuli can be interpreted as follows. At the short durations, the lines were not on the screen for a long enough time for accurate identification; the subjects could not divide adequately their attention between the four channels. The longer durations afforded better identification of the lines, but at the longest duration maintaining the memory trace became difficult, as evidenced by the drop in performance. It should be noted that the best VM detection occurred at a duration of $160 \mathrm{msec}$, even though all the training had occurred at a $100-\mathrm{msec}$ duration. The drop-off for longduration displays suggests that the memory trace of the preceding line features decayed in the time between the display of the first line and the display of the last line. The CM sequences were all identified at a similar peformance level, with the implication that these stimuli required fewer attentional resources and the memory trace decayed less quickly. The attentional demands of CM and VM stimuli fit in with the results from Experiments 2 and 3 and with the literature on letter and digit identification reviewed above. The memory-trace aspects of the CM and VM differences could be studied in the present research because of the special nature of the sequences used. The feature did not appear simultaneously on the screen and the timing characteristics of the individual features could be manipulated; letter and digit stimuli do not allow for such a control over individual features.

\section{EXPERIMENT 5 LINE FEATURE OVERLAP}

LaBerge (1976) argued that practice would allow the features that compose novel stimuli to develop automatically into a code, or unit. His data showed that early in practice, identification of the stimulus required that attention be allocated, but that late in practice, attention was not needed. A characteristic of automatization, therefore, is that the stimulus is perceived by the subject as a unit and thus that the individual features need not be identified through attention. Treisman and Gelade (1980) articulate a somewhat similar concept. They argue that the separable features that compose a stimulus are free- floating until attention provides the "glue" that combines the individual features into a whole stimulus. They do not consider, however, the changes in the amount of attention needed to "glue" the features together with practice. The Treisman and Gelade position is different from the LaBerge approach in that they claim that all stimuli composed of separable features, not just novel stimuli, require attention for the features to be "glued" into a whole. The two theoretical positions are similar in that they both imply that a stimulus is perceived differently, over some degree of unitization of the stimulus, depending on the familiarity of the stimulus (LaBerge) or whether attention is allocated to the stimulus (Treisman \& Gelade).

One problem with both of these positions is that the concept of a feature is defined poorly for the letters and digits typically studied. The sequences studied in the present series of experiments allow for better control over the features that compose a stimulus; by occurring at different times, the features can be defined and the separation of the features can be seen clearly. In particular, the unitization of the stimulus can be changed by manipulation of the overlap of the individual features. When the individual features have no overlap, as they have had in the previous experiments, then the subject could possibly perceive the stimulus as three individual lines instead of as a whole. When the lines are overlapped, the stimulus would appear to be more of a unit rather than individual lines.

In this experiment, the stimuli were artificially unitized by overlapping the individual line segments. We then tried to determine, by having subjects subjectively rate the stimuli on a feature/unitization scale, if they perceived the CM and VM stimuli differently.

\section{Method}

In this experiment, the duration of the line segments was always $100 \mathrm{msec}$. The amount of overlap was an independent variable with five different levels: $80 \%, 60 \%, 40 \%, 20 \%$, and $0 \%$. At the $80 \%$ level, the second line of the sequence occurred during $80 \%$ of the first line and the third line overlapped $80 \%$ of the second line. Looking at it another way, the second line occurred $20 \mathrm{msec}$ after the onset of the first line and the third line occurred $20 \mathrm{msec}$ after the onset of the second line. The procedure was similar, depending on the percentage of overlap, for the other levels. Stimuli in the $0 \%$ level were the same as the training stimuli. The percent overlap was crossed with the stimulus group, CM or VM, to produce 10 different kinds of blocks of trials. A block consisted of eight trials in which the overlap and target group (CM and VM) were the same for all trials of the block. The subjects were required to identify the target. At the end of the eight trials, the subjects wrote down their ratings for the trials of the block. They were instructed that a rating of " 1 " meant that the lines formed a solid figure and a " 7 " meant that the line pattern appeared to be a single line moving around. This experiment consisted of 20 blocks and lasted about $30 \mathrm{~min}$. Before this experiment, the subjects had about $2 \mathrm{~h}$ of practice in identifying the overlapped stimuli so that they were very familiar with all the stimuli they were to rate.

\section{Results and Discussion}

The ratings for the $\mathrm{CM}$ and $\mathrm{VM}$ sequences are presented in Figure 9. A repeated measures three-way ANOVA, with overlap being the repeated measure and subjects the 


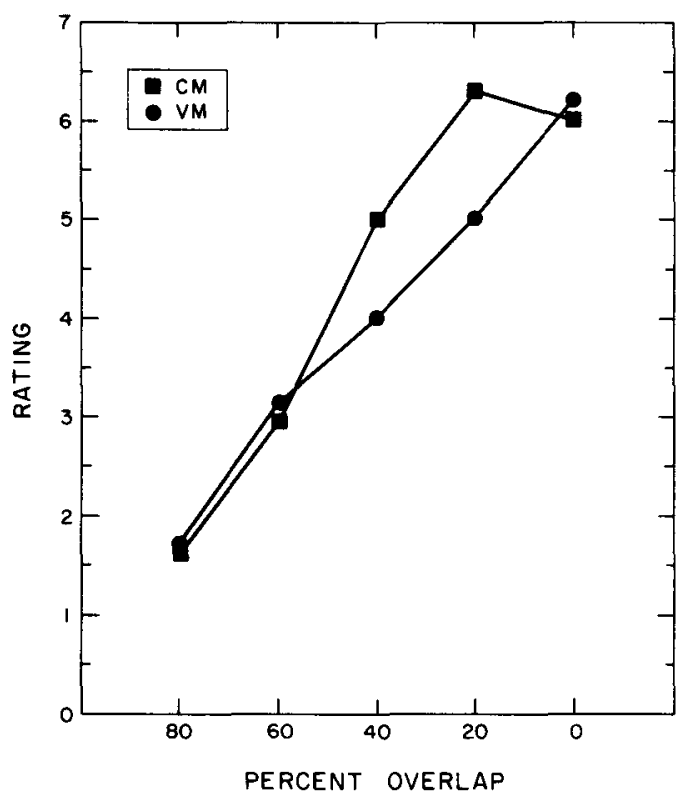

Figure 9. The ratings score as a function of the percent overlap of the line segments from Experiment 5. A "1" means that the pattern was figure-like and a " 7 " means that the pattern looked like a single line moving around.

random factor, was performed on the data. The ratings differed as a function of the percent overlap $[F(4,24)=$ $40.67, p<.001$ ], which means that subjects followed instructions and used the whole range of ratings. The difference between CM and VM sequences was nonsignificant. The most important part of the data is the significant interaction between stimulus group (CM or VM) and the percent overlap $[F(4,24)=4.03, p<.05]$. The VM sequence ratings appear to be a linear function of the percentage of overlap. The CM rating is different, though, in that it has a bump in the $0 \%-40 \%$ overlap range that is not present in the VM ratings. The $20 \%$ overlap rating is about equal to the $0 \%$ rating in the $\mathrm{CM}$ condition.

The significant interaction suggests that the $\mathrm{CM}$ sequences were perceived differently from the VM sequences along some dimension of features/unitization, at least for the sequences that were similar to the ones the subjects were trained on. For the CM stimuli, the $0 \%$ and $20 \%$ overlap sequences were rated similarly. In effect, the line segments of the CM sequences can be overlapped for $20 \%$ of the duration of the segments and the subjects report that they perceive no difference between those and the $0 \%$ overlap sequences. The interaction cannot be accounted for by a change in criterion for the VM and CM groups because the same subjects rated both groups.

\section{EXPERIMENT 6 ROTATION}

The design of this experiment was motivated by the results of Experiment 4. In Experiment 4, we found high transfer from the trained stimuli to the novel stimuli when the line durations were manipulated. The subjects had never before seen those particular stimuli, whose line durations were shortened or lengthened from $100 \mathrm{msec}$. Under what other conditions could we expect transfer to new stimuli to remain high? Experiment 4 manipulated the temporal component of the stimulus. In this experiment, the spatial component was manipulated by rotating the stimuli and the transfer to these novel stimuli was evaluated.

\section{Method}

The one-sequence display similar to that used in the first part of Experiment 1 and in Figure 2 was used. The independent variable of importance was the amount of rotation. Each sequence was rotated $0^{\circ}, 90^{\circ}, 180^{\circ}$, or $270^{\circ}$; the orientation target sequence was also rotated. The $0^{\circ}$ condition was exactly the same as the procedure used for Experiment 1.

Rotation was a between-block variable. Each block contained eight repetitions of each of the eight VM targets and eight repetitions of the CM targets, for 72 trials altogether. In this experiment, the subjects participated for a total of 12 blocks, three repetitions of each of four rotation conditions. The experiment lasted approximately $2 \mathrm{~h}$.

\section{Results and Discussion}

A repeated measures three-way ANOVA, with subjects as the random factor, was performed on the correctedfor-guessing percentage scores after an arcsin transformation (see Figure 10). The interaction between stimulus group (CM or VM) and degrees of rotation was significant $[F(3,18)=4.17, p<.025]$. Significant main effects also occurred for the degrees of rotation $[F(3,18)$ $=5.41, p<.01]$ and for the stimulus group (CM or VM) $[F(1,6)=7.21, p<.05]$. Because of the significant interaction, though, these significant main effects have to be interpreted with caution. By looking at the simple main effects of stimulus group at each of the four rotation levels, only at the $0^{\circ}$ rotation were the CM stimuli detected better than the VM stimuli $[F(1,6)=55.38, p<.001]$. At the other three rotation levels, no difference between $\mathrm{CM}$

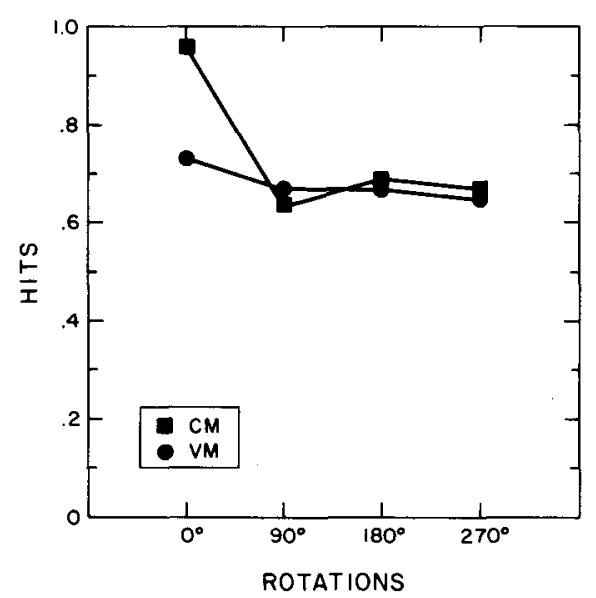

Figure 10. The proportion of trials identified correctly as a function of the amount of rotation in Experiment 6. The hits are corrected for guessing. 
and VM stimuli was found. The significant interaction indicates that when the CM sequences are rotated, performance falls to a level that could be expected for novel sequences. VM performance also drops, but not as much as $\mathrm{CM}$ performance.

\section{OVERALL DISCUSSION AND CONCLUSIONS}

This series of experiments had two related purposes. First, we were interested in determining whether or not visual sequential stimuli exhibited automaticity characteristics similar to those seen in such simpler visual stimuli as letters and digits. Experiments 1-3 evaluated automaticity using methods used in other search and detection experiments. Second, we were interested in determining the effects of $\mathrm{CM}$ and VM practice when the individual features and the whole stimulus were manipulated. Experiments 4-6 manipulated the individual durations of the features, the overlap of the features, and the orientation of the whole stimulus. These kinds of manipulations had not been performed previously for CM and VM stimuli; the nature of the sequences allowed us to make these kinds of manipulations. These manipulations helped us to better understand the nature of automaticity and CM and VM training.

As discussed by Jonides et al. (1985), several different techniques have been used to measure automaticity. The evaluation techniques should be based ultimately on theoretical justifications for the empirical measurements used. Shiffrin and Schneider's (1977) model suggests that three characteristics are important in the evaluation of an automatic process: (1) CM performance should be superior to VM performance and should improve at a faster rate; (2) CM performance should be insensitive to changes in the display size or the number of visual channels, but VM performance should drop as the number of channels is increased; and (3) CM performance should be insensitive to changes in the target set size, but VM performance should decrease as the target set size is increased.

The differences between the CM and VM groups, the first automaticity criterion, were evaluated in Experiments 1 and 2. In Experiment 1, both the CM and VM groups improved with practice, with the $\mathrm{CM}$ performance always being superior and improving at a faster rate. This result was similar to that seen in Shiffrin and Schneider (1977). In Experiment 2, CM stimuli were detected marginally better than VM stimuli.

The effect of the number of channels, the second criterion, was evaluated in Experiment 2. Both CM and VM groups showed decreases in performance with an increase in the number of channels. In contrast to Schneider and Shiffrin's (1977, Experiment 2) findings, a decrease in performance with increasing channel size has been found in CM search with confusable stimuli (Foyle, 1981).

The effect of target set size, the third criterion, was evaluated in Experiment 3. Both CM performance and VM performance were sensitive to memory load, a result that differed from that obtained by Schneider and Fisk (1982a), who found only the VM performance to be sensitive to memory load. Similar to the earlier results, however, CM search imposed much less load on memory than did VM search. Two of the subjects performed poorly in CM dual-task detection. When evaluated separately, the remaining 5 subjects exhibited characteristics similar to those of Schneider and Fisk's (1982a) subjects.

When compared with previous experiments and the automaticity characteristics usually shown for letter and digit stimuli, these results demonstrate a weak case for automatic processing for the $\mathrm{CM}$ group. In other experiments using efficient training techniques in our laboratory (Schneider \& Shiffrin, 1977; Schneider \& Fisk, 1982a, 1982b), we have found that automatic responses to letters or digits can be trained typically in 3-5 h. After over $17 \mathrm{~h}$ of training in the present set of experiments, subjects could still not achieve the same level of performance (i.e., no decrement in performance when the number of channels increased and little or no resource consumption for CM targets when target set was changed) as that found in the other experiments. Most of the results were, however, in the direction of these previous ones. CM/VM differences were usually found and the CM single/dual decrement for 5 of the subjects was similar to or less than the decrement of individual subjects in the Schneider and Fisk (1982a) experiment.

What are some of the possible reasons for the weaker automatic processing of $\mathrm{CM}$ sequences? The sequences differed from the letters and digits previously studied in three ways: the sequences were highly confusable with each other, a memory trace of the line segment features had to be maintained for identification to occur, and the features of the stimulus did not overlap in time. Experiments 4 and 5 were designed to evaluate the CM/VM differences in terms of these characteristics of the stimuli. Each of these factors and their relationships to Experiments 4 and 5 will be discussed.

First, the sequences were highly confusable with each other. All the lines were the same length and differed only in orientation and position. None of the other stimulus sets previously studied and mentioned in this article had stimuli in which the features were all the same. The LaBerge (1976) work earlier showed that the development of automatic responses to novel stimuli composed of lines was difficult. A number of other experiments that used visually confusable stimuli (e.g., Foyle, 1981; Shiffrin \& Dumais, 1981) have shown similar attentional effects after CM and VM training. Foyle examined $\mathrm{CM}$ and VM search for letters and found that searching for highly confusable letters remained serial and resource consumptive even after extensive training. Schneider and Eberts (1980) examined color and shape conjunction search and found that $\mathrm{CM}$ practice at shape and conjunction search resulted in substantial performance improvement but that the search remained serial and resource consumptive. This suggests that automatic detection is limited to situations in which the target and distractor sets are easily discriminable. In the present study, movement patterns were 
noncontinuous rather than simple movements (e.g., reversals and transversals). We speculate that if we had used the simplest movement patterns, such as rotation or unidirectional movement (Eberts, 1979), we would have replicated the earlier results of CM performance insensitivity to increasing channel size.

Second, subjects had to maintain a memory trace of the first two line segments if the whole sequence was to be identified. Unlike letters and digits, all the features were not displayed on the screen at the same time for the sequences. Maintaining the memory trace could also require some attentional resources for both $\mathrm{CM}$ and VM sequences. When the length of time that the memory trace had to be maintained was manipulated in Experiment 4, $\mathrm{CM} / \mathrm{VM}$ differences occurred. Performance dropped off at the longest line durations for the VM stimuli, which implied that the memory trace for the early-occurring features could have been lost. No drop-off in performance was seen for the CM sequences, which implies that the memory trace was easier to maintain.

Third, the features of the whole stimulus did not overlap in time. To identify the sequences, subjects had to try to perceive the three line segments and then determine how these fit together as a whole. Remembering the three line segments when not displayed on the screen could have required attentional resources and could have accounted for the decrease in performance for both CM and VM sequences when the number of channels was increased in Experiment 2. Experiment 5 offered evidence, however, that CM and VM sequences were perceived differently in terms of a feature/unitization criterion. Subjects reported that they perceived the $0 \%$ and $20 \%$ overlapped CM sequences to be similar to each other, even though the $20 \%$ overlap caused the stimulus to appear more holistic. This pattern was not seen for the VM sequences.

Experiment 4 showed that high transfer could occur to novel stimuli if the line durations were manipulated, even though the pattern of results was different for $\mathrm{CM}$ and VM stimuli. In data not reported in this article, when performance measures were taken for the overlapped stimuli of Experiment 5, high transfer to the novel overlapped stimuli was also found. These two experiments varied the timing characteristics of the line segments. Experiment 6 manipulated the rotation of the sequences to determine if high transfer occurred for these novel rotated stimuli. Rotating the stimuli eliminated the CM advantage. The significant interaction between the stimulus group and the amount of rotation indicated that the two stimulus groups were differentially affected by this manipulation. In particular, the VM stimuli were relatively unaffected in that performance dropped by only $11 \%$ when rotated. Rotation had a larger effect on the CM stimuli in that performance dropped by $33 \%$ when the rotated stimuli were compared with the unrotated stimulus. Putting these decrements into perspective: $\mathrm{CM}$ performance regressed to a level similar to that during the second hour of training and VM performance regressed to a level at some time after $7 \mathrm{~h}$ of training.

In summary, the CM sequences studied in these experiments exhibited only weak indications of automatic detection and processing when compared with previous experiments on letter and digit stimuli. Due to the nature of sequential stimuli, the manipulations that could be performed, which differed from those used in previous experiments, expanded our knowledge about the automatic processing of sequential stimuli and the effects of $\mathrm{CM}$ training. In particular, the memory trace was more robust for $\mathrm{CM}$ sequences than for $\mathrm{VM}$ sequences, $\mathrm{CM}$ sequences were perceived differently from VM sequences on a feature/unitization scale, and rotation of the $C M$ sequences destroyed the performance advantages the $\mathrm{CM}$ sequences had had over the VM sequences.

These experiments illustrate that extended practice alone is not enough to produce the parallel, low-resource, difficult-to-control processing associated with automatic letter and category search. One must also consider the type and confusability of stimuli. Schneider and Mummy (1986) have illustrated how a quasi-neural simulation model develops automatic processing. This model illustrates the observed results that stimuli that are difficult to discriminate are less likely to develop automatic processing. In an environment in which the stimuli are generally easily discriminable, presented well above threshold, and well practiced, automatic processing would be expected. However, the present complex noncontinuous movements were difficult to discriminate (fouralternative force-choice accuracy was below $50 \%$ for the first 320 trials). The processing of these stimuli improved, but they still required substantial attentional resources, even with the consistent practice.

\section{REFERENCES}

Bushbaum, W. H., \& Mayzner, M. S. (1969). The effect of line length on sequential blanking. Psychonomic Science, 15, 111-112.

Craig, A. (1979). Nonparametric measures of sensory efficiency for sustained monitoring tasks. Human Factors, 21, 69-78.

DeSilva, H. R. (1926). An experimental investigation of the determinants of apparent visual movement. American Journal of Psychology, 37, 469-501.

EBERTS, R. E. (1979). The automatic and controlled processing of sequences of events (Tech. Rep. No. 7901). Champaign, IL: University of Illinois, Human Attention Research Laboratory.

ERIKSEN, B. A., \& ERIKSEN, C. W. (1974). Effects of noise letters upon the identification of a target letter in a nonsearch task. Perception \& Psychophysics, 16, 143-149.

Fehrer, E., \& RAAB, D. (1962). Reaction time to stimuli masked by metacontrast. Journal of Experimental Psychology, 63, 143-147.

Fisk, A. D., \& SChNeider, W. (1983). Category and word search: Generalizing search principles to complex processing. Journal of Experimental Psychology: Learning, Memory, \& Cognition, 9, 177-195.

FOYLE, D. L. (1981). Automatic processing and selective attention. Unpublished doctoral dissertation, Indiana University, Psychology Department.

Graham, C. H. (1965). Perception of movement. In C. H. Graham (Ed.), Vision and visual perception. New York: Wiley.

Jonides, J., Naveh-Benjamin, M., \& Palmer, J. (1985). Assessing automaticity. Acta Psychologica, 60, 157-171. 
Kahneman, D. (1967). An onset-onset law for one case of apparent motion and metacontrast. Perception \& Psychophysics, 2, 577-584.

Kahneman, D., \& Wolman, R. E. (1970). Stroboscopic motion: Effects of duration and interval. Perception \& Psychophysics, 8, 161-164.

Kolers, P. A. (1964). The illusion of movement. Scientific American, 211(4), 98-106.

LABERGE, D. (1973). Identification of two components of the time to switch attention: A test of a serial and a parallel model of attention. In S. Komblum (Ed.), Attention and performance $I V$ (pp. 71-85). New York: Academic Press.

LaBerge, D. (1976). Perceptual learning and attention. In W. K. Estes (Ed.), Handbook of learning and cognitive processes (Vol. 4, pp. 237273). Hillsdale, NJ: Erlbaum.

NeISSER, U., \& BECKLEN, R. (1975). Selective looking: Attending to visually specified events. Cognitive Psychology, 7, 480-494.

NORMAN, D. A. (1964). A comparison of data obtained under different false-alarm rates. Psychological Review, 71, 243-246.

Ostry, D., Moray, N., \& MARKS, G. (1976). Attention, practice, and semantic targets. Journal of Experimental Psychology: Human Perception \& Performance, 3, 326-336.

Poltock, S. E., Lansman, M., \& Hunt, E. (1981). Automatic and controlled processing in auditory detection (Tech. Rep. No. 9). Seattle: University of Washington, Psychology Department.

RESTLE, F. (1979). Coding theory of the perception of motion configuration. Psychological Review, 86, 1-24.

SCHNEIDER, W. (1985). Toward a model of attention and automatic processing. In M. I. Posner \& O. M. Morin (Eds.), Attention and performance $X I$ (pp. 475-492). Hillsdale, NJ: Erlbaum.

SCHNEIDER, W., \& MUMMY, D. (1986). Attention and automaticity and the compiling of knowledge: A two level architecture. Manuscript in preparation. Pittsburgh, PA: University of Pittsburgh Learning Research \& Development Center.
SCHNEIDER, W., \& EberTs, R. E. (1980). Automatic processing and the unitization of two features (Report No. 8008). Champaign, IL: University of Illinois, Psychology Department.

SChNeider, W., \& Fisk, A. D. (1982a). Concurrent automatic and controlled visual search: Can processing occur without resource cost? Journal of Experimental Psychology: Learning, Memory, \& Cognition, 8, 261-278.

SCHNEIDER, W., \& FiSK, A. D. (1982b). Degree of consistent training: Improvements in search performance and automatic process development. Perception \& Psychophysics, 31, 160-168.

SCHNEIDER, W., \& FISK, A. D. (1984). Automatic category search and its transfer: Automatic process semantic filtering. Journal of Experimental Psychology: Learning, Memory, \& Cognition, 10, 1-15. SCHNeIder, W., \& Shiffrin, R. M. (1977). Controlled and automatic human information processing: I. Detection, search and attention. Psychological Review, 84, 1-66.

Shiffrin, R. M., \& Dumais, S. T. (1981). The development of automatism. In J. R. Anderson (Ed.), Cognitive skills and their acquisition (pp. 111-140). Hillsdale, NJ: Erlbaum.

Shiffrin, R. M., \& SCHNEIDER, W. (1977). Controlled and automatic human information processing: II. Perceptual learning, automatic attending and a general theory. Psychological Review, 84, 127-190.

Treisman, A. M., \& Gelade, G. (1980). A feature-integration theory of attention. Cognitive Psychology, 12, 97-136.

Werthelmer, M. (1912). Experimentelle Studien über das Sehen von Bewegung. Zeitschrift für Psychologie, 61, 161-265.

(Manuscript received November 6, 1985; revision accepted for publication April 7, 1986.) 